\title{
Career choices and global health engagement: 24-year follow-up of U.S. participants in the Indiana University-Moi University elective
}

Rachel A. Umoren, MBBCh, MS,,${ }^{1,4}$ Adrian Gardner, MD, MPH, ${ }^{2,3}$ Geren S. Stone, MD, DTM\&H, ${ }^{5,6}$ Jill Helphinstine, MD, ${ }^{7}$ Emily P. Machogu, MD, ${ }^{2,7}$ Jordan C. Huskins, MD, ${ }^{7}$ Cynthia S. Johnson, ${ }^{8}$ Paul O. Ayuo, MBChB, MMed, DLSHTM, MSc, ${ }^{3}$ Simeon Mining DVM, MSc, PhD, ${ }^{3}$ Debra K. Litzelman, MD ${ }^{1,2,9}$

${ }^{1}$ Center for Health Services Research, Regenstrief Institute, Inc., Indianapolis, IN

${ }^{2}$ Department of Medicine, Indiana University School of Medicine, Indianapolis, IN

${ }^{3}$ Moi University School of Medicine, Eldoret, Kenya.

${ }^{4}$ Department of Pediatrics, University of Washington School of Medicine, Seattle, WA

${ }^{5}$ Global Primary Care (GPC) Program, Center for Global Health, Division of General Medicine, Department of Medicine, Department of Medicine, Massachusetts General Hospital, Boston, MA

${ }^{6}$ Harvard Medical School, Boston, MA

${ }^{7}$ Department of Pediatrics, Indiana University School of Medicine, Indianapolis, IN

${ }^{8}$ Department of Biostatistics, Indiana University, Indianapolis, IN

${ }^{9}$ Indiana University Center for Global Health, Indianapolis, IN

Running Title: Long-term career choices of global health learners

Corresponding author: Rachel A. Umoren, 1959 NE Pacific Street, Box 356320, Seattle, WA 98195. Email: rumoren@uw.edu

Key Words: medical education; global health electives; residents; career choice; medical practice

This is the author's manuscript of the article published in final edited form as:

Umoren, R. A., Gardner, A., Stone, G. S., Helphinstine, J., Machogu, E. P., Huskins, J. C., ... Litzelman, D. K. (2015). Career choices and global health engagement: 24-year follow-up of U.S. participants in the Indiana University-Moi University elective. Healthcare, 3(4), 185-189. http://doi.org/10.1016/j.hjdsi.2015.10.001 
In 1989, the Indiana University School of Medicine (IUSM) formed an academic partnership with Moi University (MU) in Eldoret, Kenya. The partnership was formed for mutual institutional benefit with the following goals in mind: to assist in delivery of health care services, develop leaders in health care for both the United States and Kenya, and conduct health research. Although the partnership began as an individual institution partnership, it was expanded in 1997, with other academic institutions in the U.S. and Canada joining the work of IUSM in Kenya and establishing a consortium of universities called the Academic Model Providing Access to Healthcare (AMPATH). In reports on the bilateral faculty and learner exchange, trainees often speak of their experiences in terms of "life-changing," "challenging," "incredible," and “enlightening." $1-4$

Educators investigating the short-term benefits of global health electives have reported increased knowledge of preventive medicine and public health; ${ }^{5}$ increased competency in physical examination and procedure skills; ${ }^{6}$ a greater likelihood of matching to primary care residencies; ${ }^{7}$ with trends toward increased resource efficiency, ${ }^{8-14}$ cultural competence, and a global world view. ${ }^{15,16}$ These findings reflect the opportunities that global health electives provide for personal and professional development in patient care and communication skills, systems based practice, practice based learning and improvement, and professionalism. ${ }^{17-18}$

There are few long-term studies of outcomes of global health experiences. The University of Wisconsin international health fellowship program surveyed 42 medical student participants 4-7 years after participation, and found that they were more likely to work with underserved populations and engage in community service activities. ${ }^{10}$ The Duke University Medicine Residency Program surveyed 59 resident participants and 138 nonparticipants up to 7 years after participation showing that residents who changed career plans during residency tended to move 
towards general internal medicine or public health. ${ }^{11}$ A Yale University survey of 96 internal medicine residents who participated in an international health program at U.S. and international sites from 1982 to 1996, showed similar trends towards primary care and care for the underserved compared with nonparticipants. ${ }^{19}$ The studies represent a growing body of evidence of the impact of global health experiences. To our knowledge there are no long-term follow up studies that involve medical students and residents from multiple residency programs who participated in a global health elective at a single international site.

\section{Methods}

The IUSM-Moi elective has grown to host approximately 35-40 medical students and residents from multiple residencies including Internal Medicine, Pediatrics, Obstetrics and Gynecology and combined programs such as Internal Medicine-Pediatrics, Emergency Medicine-Pediatrics, and Pedatrics-Psychiatry-Child Psychiatry each year. In nearly a quarter of a century of partnership with the Kenyan institution, an estimated 560 medical trainees have completed the two-month long global health elective (GHE) with supervised inpatient rotations at Moi Teaching and Referral Hospital which is the teaching hospital for Moi University School of Medicine (MUSM), community health experiences, and weekly opportunities for discussion and reflection (fireside chats) conducted by onsite IUSM faculty.

We designed the follow up study as a cross sectional survey with study and control groups. The survey instrument was developed by study investigators with the input of global health experts and contained items on current medical practice and engagement in primary care, public health, global health, and care of underserved and diverse populations. For past participants, the survey 
included 38 items and included a series of questions about demographics, dates of rotation(s) in Kenya, stage in training at the time of the elective, current clinical practice, awareness of the cost of care, current engagement with local and global health activities, value of international electives in medical education, and the perceived influence of the GHE. The control group similarly received an abbreviated 23-item survey without questions specific to the GHE, but which included questions on barriers to participation. The study was approved as exempt from further review by the Indiana University Institutional Review Board.

\section{Study Participants}

\section{Global Health Elective (GHE) group}

The GHE group consisted of IUSM alumni who were identified from program records as participating in the IUSM-Moi elective.

\section{Inclusion criteria}

Participation in the IUSM-Moi elective as a medical student and/or resident between January 1989 and May 2013.

\section{Exclusion criteria}

Due to the focus of the survey on long-term career choices and clinical practice behaviors, any past participant who was currently a student or resident was excluded from the study.

\section{Control group}

We selected a random sample of IUSM alumni who graduated from 1989-2013 from the alumni database, with the number of invitations sent to controls stratified by year of graduation in order to account for the increasing number of GHE participants over the study period. 
Study procedures: Investigators gathered contact information including work or home addresses, email, or phone number for former participants in the GH elective. The information was obtained from the university partnership records, the Indiana University Alumni Association (IUAA), and searches of social media sites (Facebook and Linked-In). From October to December 2013, we contacted eligible individuals in the GHE group up to three times by email, inviting them to participate in an online survey using REDCap. ${ }^{20}$ A reminder was sent approximately one month following the initial mailing and followed by a second survey for all non-respondents. Emailed invitations contained personalized survey links for tracking purposes. We sent non-respondents with postal addresses a survey with a link for online completion and a pre-paid return envelope via postal mail. From December 2013 to March 2014, we contacted a random sample of non-participants identified through the IUAA up to three times by email. The survey was closed after a comparable number of GHE and control group responses were obtained.

\section{Statistical methods}

We used Chi-squared tests to compare groups (participants vs. non-participants) on categorical variables. When there was an overall significance, pair-wise comparisons were done using Hochberg's step-up Bonferroni adjustment for multiple comparisons. Mantel-Haenszel chisquare tests were used to compare groups on ordinal variables. Two-sample t-tests were used to compare continuous variables between the groups. All variables significant at the 0.25 level on univariate analysis were included in multivariable logistic regression analysis. Backwards elimination was used to determine the final model. All variables significant at the 0.05 level of significance were retained in the final model. Due to small sample sizes, some categories on 
scales were combined in the multivariable analysis. Hosmer and Lemewshow goodness of fit test was done to assess the fit of the multivariable model. The same tests were used to compare individuals within the GHE group who participated in multiple electives at Moi University and individuals with a single elective, but Fisher's exact tests were used in place of chi-squared tests when expected cell sizes were less than five.

\section{Results}

We obtained current contact information for 396 individuals (about 70\% of the estimated 566 individuals who participated in the elective). A total of 203 individuals in the GHE group responded $($ response rate $=51 \%)$. After excluding ineligible and incomplete responses, a total of 176 responses from previous participants in the $\mathrm{GH}$ elective were included in the analysis. A total of 1076 survey invitations were sent to non-participants; 217 responded prior to study closure. After excluding ineligible and incomplete responses, a total of 177 respondents from the control group were included in the analysis. (Figure 1)

[Insert Figure 1. Survey respondents in Global Health Elective (GHE) and Control groups]

\section{Demographics of survey respondents}

Age, gender, and ethnicity were similar between the GHE group and control groups (Table 1). In the GHE group, 120 respondents were medical students and 56 were residents at the time of the elective. The responders and non-responders in the GHE group were also similar for age 
$(p=0.88)$, gender $(p=0.88)$, ethnicity $(p=0.12)$, and status as student or resident at the time of the elective $(\mathrm{p}=0.34)$.

[Insert Table 1. Demographics of survey respondents]

\section{Career choices}

There was a significant difference between type of practice between the GHE and control groups $(p=0.003)$. Pair-wise comparisons indicated that study group participants were more likely to be primary care providers or hospitalists compared to subspecialists. Participants reported practicing in a wide range of primary care or hospitalist fields after graduation, including: general internal medicine, general pediatrics, family medicine, combined medicine/pediatrics, and obstetrics and gynecology. These findings support the recent report by Bruno et al., ${ }^{7}$ that showed that GHE medical student participants were more likely to match to primary care residencies. In addition, they provide evidence that GHE participants are less likely to enter fellowships after residency and tend remain in primary care. There were no significant differences between groups in time spent in academic medicine or providing direct patient care. However, practice location significantly differed between the groups: pair-wise tests indicated that GHE group respondents were more likely than the control group to practice internationally than in rural, urban or suburban settings in the U.S. (Table 2).

[Insert Table 2. Univariate group comparisons of practice type and location here]

\section{Current engagement with local or international global health}


GHE group participants spent more time in global health, public health, public policy or health services research activities than did controls ( $\mathrm{p}=0.005$ ). In the GHE group, $9 \%$ of 176 respondents spent at least half their time in these activities compared to $3 \%$ of 177 controls. GHE participants also rated their involvement in local or global advocacy higher than controls $(\mathrm{p}=0.001)$. We asked respondents to indicate if they participated in short-term trips $<1$ week per year or routinely worked outside the U.S for longer periods of time. GHE respondents were more likely to spend part of the year providing healthcare internationally with $10 \%$ of $176 \mathrm{GHE}$ respondents providing international care for greater than 3 weeks per year, compared to $0.6 \%$ of 177 controls (Table 2).

\section{Awareness of the cost of medical care}

By their self-report, awareness of the cost of medical care was more likely to affect the choices of GHE participants compared to controls ( $\mathrm{p}<0.001)$. A total of $167(95 \%)$ of the GHE respondents stated that the cost of medical care either occasionally or often affects their choices compared to 153 (87\%) of controls. Additionally, GHE participants were asked to rate, on a scale of 1 to 100 , the influence of the MU elective on their awareness of the cost of medical care. The mean (SD) score was 68.3 (23.1) and the median score was 70 . Only $10 \%$ of respondents gave a score less than 40 and $18 \%$ of respondents gave a score above 90.

\section{Providing care to underserved populations}

GHE participants were also more likely to provide care to underserved populations in the U.S. $(p=0.04)$ (See Figure 2). However, they were not more likely than the control group to report 
practice in rural areas, that their patient population was very diverse, or that they routinely delivered cross-cultural care. While only $6 \%$ of the GHE group participated in more than one rotation, these individuals were more likely that those with a single rotation to identify their patient population as underserved ( $\mathrm{p}=0.04$ ), to participate in healthcare outside the U.S. $(\mathrm{p}<0.001)$, spend less time in direct patient care $(\mathrm{p}=0.001)$, allocate more of their work week to academic medicine $(\mathrm{p}=0.02)$, and dedicate more of their time to global or public health and related activities $(\mathrm{p}<0.001)$.

[Insert Figure 2. Comparison of respondents' estimated percentage of underserved patients]

\section{Multivariable group comparisons}

The three factors that remained significantly associated with GHE participants after multivariable analysis were: type of practice, influence of the awareness of healthcare costs on medical decision-making, and ongoing provision of healthcare outside the U.S. ( $p=0.01, p=0.03$, and $\mathrm{p}=0.001$, respectively). GHE participants had 2.2 times the odds of being hospitalists vs. subspecialists and 1.8 times the odds of being primary care providers vs. subspecialists when compared with the control group. Providers whose choices were at least occasionally affected by cost awareness had 2.7 times the odds of being GHE participants than controls. GHE participants had 4.9 times the odds of providing healthcare outside the U.S. $\geq 1$ week per year. (Table 3)

[Insert Table 3. Results of Multivariable Analysis] 


\section{Discussion}

The Lancet Commission on Education of Healthcare Professionals for the 21 st Century ${ }^{21}$ called for the development of innovative educational programs that encourage increased respect and compassion for the underserved with an enhanced commitment to primary health care needs. Physicians in the GHE group are more likely to make choices that reflect these values. While global health electives may not be causally related to an individuals' decision to enter a primary care field or care for underserved populations, the association between these experiences and long-term career choices in these areas supports the benefits of greater integration of these experiences into health professional training.

Finding ways to encourage physicians to think about how the decisions they make contribute to patient or societal costs is increasingly important. The Lancet Commission on Education of Healthcare Professionals for the 21 st Century $^{21}$ also called for ways to cultivate the formation of a new health care professional with an internalized social accountability for limited healthcare resources. There is a need to implement innovative methods that teach practicing physicians and trainees how to integrate cost considerations into everyday care. ${ }^{22-24} \mathrm{We}$ found that GHE are associated with a long-term cost-consciousness, an attitude that participants can apply in their local medical practice. While it may not be possible for every learner to participate in a global heath elective, it is possible that local service learning experiences or immersive 3D global health scenarios ${ }^{25}$ may serve as a supplement to training and/or provide a proxy global health experience with similar positive impact on trainees' eventual practice and cost effective medical decision making. 
Regardless of their location, underserved populations may have comparable needs. Providers with similar professional goals and values may be best equipped to address these needs in various contexts. The GHE group was significantly more likely to report providing care to underserved populations than the control group. These findings are supported by other studies in which participants in global health tracks were more likely than non-participants to care for underserved populations in the U.S. including those in health professional shortage areas and areas of dense poverty. ${ }^{26,27}$ Global health tracks provide a more intensive exposure to global health issues along an immersive global health experience, and it is encouraging that the outcomes of graduates with short-term global health experiences may be similar despite the shorter exposure.

On the other hand, GHE participants were not more likely than the control group to practice in rural areas. These findings differ from previous reports on family medicine participants in a global health track who were more likely to practice in rural areas than non-participants. ${ }^{26}$ It is possible that the increased likelihood of the GHE group to be hospitalists may contribute to this finding as fewer hospitalists work in rural areas. Other studies show that global health experiences are linked to self-reported increases in cultural competence. ${ }^{15,16}$ While in our study, GHE participants were not more likely than the control group to report delivering cross-cultural care; this finding may be due to the increasing opportunity for all physicians to do so. It is also possible that global health programs may attract a different type of learner, focused on very different types of cross-cultural experiences, than those obtainable in even highly diverse U.S. settings. 
The subgroup of study participants that had multiple rotations during their training was small. However, individuals that participated in more than one global health rotation were significantly more likely to participate in healthcare outside the U.S., practice academic medicine and identify their patient population as underserved when compared to those with a single experience. These findings are comparable to those reported on participants of global health tracks. ${ }^{26,27}$ The impact of long-term engagement in global health activities and providing health care outside the U.S. after graduation is an area needing further study as this may renew or enhance the positive elements associated with global health experiences.

\section{Study limitations}

This was an international long-term follow up study of GHE past participants and alumni. While our study results support associations, individuals interested in global health are a self-selected group; so it is difficult to establish the true role of these experiences in career choice and practice. One of the limitations was that while surveys were sent to all individuals who indicated interest in the elective, program records included learners that were not able to travel to Kenya for various reasons. It is possible that individuals in the latter group failed to respond to the survey contributing to the relatively low response rate. The response rate for the control group could not be determined because the survey was closed after we had received a similar number of responses to the GHE group. While the low response rate may introduce response bias, there were no significant differences between the demographics of respondents and non-respondents in the GHE group and between the GHE and control groups. Further studies that prospectively examine the impact of early and multiple global health experiences during and prior to medical school are needed to define the impact of these experiences. 


\section{Conclusions}

Our long-term follow up of participants in the GH elective showed that respondents in the GHE group were more likely than controls to be generalists working with underserved populations, to report that an awareness of the cost of care affected their medical decision-making, and to report ongoing involvement in global health, public health or public policy. These findings add to the growing body of evidence that individuals who are interested in global health experiences contribute to the U.S. primary care workforce and display cost-conscious attitudes that are essential to the evolution of medicine in the U.S. The long term findings on career choices support trainee interests reported in short-term studies and indicate that cost-conscious attitudes may persist over time in global health-interested individuals. In the context of a primary care provider shortage and need for greater cost-conscious medicine, evidence is building that global health experiences may yield broader benefits to the U.S. medical system.

Acknowledgements: This study was internally funded through a grant from the Dexter Fellowship Program. One of the authors (DKL) received grant support for this work from the National Institutes of Health-NIAMS 5R25AR060994. We would like to thank the Indiana University Center for Global Health and the alumni that participated in this study.

\section{Conflict of Interest:}

Dr. Umoren declares no conflict of interest

Dr. Gardner declares no conflict of interest

Dr. Stone declares no conflict of interest

Dr. Helphinstine declares no conflict of interest

Dr. Machogu declares no conflict of interest

Dr. Huskins declares no conflict of interest

Ms. Johnson declares no conflict of interest

Dr. Simeon Mining declares no conflict of interest

Dr. Paul Ayuo declares no conflict of interest

Dr. Litzelman declares no conflict of interest 


\section{References}

1. Einterz RM, Kelley CR, Mamlin JJ, Van Reken D. Partnerships in international health. The Indiana University-Moi University experience. Infectious disease clinics of North America. 1995;9:453.

2. Oman K, Khwa-Otsyula B, Majoor G, Einterz R, Wasteson A. Working collaboratively to support medical education in developing countries: the case of the Friends of Moi University Faculty of Health Sciences. Educ Health (Abingdon). 2007;20:12.

3. Umoren RA, Einterz RM, Litzelman DK, Pettigrew RK, Ayaya SO, Liechty EA. Fostering reciprocity in global health partnerships through a structured, hands-on experience for visiting postgraduate medical trainees. Journal of Graduate Medical Education. 2014;6:320-325.

4. Huskins J, Owiti P, Wambui C, Stone G, Umoren, R, Helphinstine, J, Litzelman DK, Mining S. Ayuo P, Gardner A. Ulienda wapi: Long-term follow-up of past participants of North American and European rotations from Moi University School of Medicine, Kenya. Annals of Global Health. 2014;80:164.

5. Bjorklund AB, Cook BA, Hendel-Paterson BR, Walker PF, Stauffer WM, Boulware DR. Impact of global health residency training on medical knowledge of immigrant health. Am J Trop Med Hyg. 2011;85:405-408.

6. Hau DK, Dipace JI, Peck RN, Johnson WD, Jr. Global health training during residency: the Weill Cornell Tanzania experience. J Grad Med Educ. 2011;3:421-424.

7. Bruno D, Imperato P, Szarek M. The Correlation between global health experiences in low-income countries on choice of primary care residencies for graduates of an urban US medical school. J Urban Health. 2014;91:394-402.

8. Oliphant JL, Ruhlandt RR, Sherman SR, Schlatter MG, Green JA. Do international rotations make surgical residents more resource-efficient? A preliminary study. Journal of surgical education. 2012;69:311-319.

9. Waddell WH, Kelley PR, Suter E, Levit E. Effectiveness of an international health elective as measured by NBME Part II. Academic Medicine. 1976;51:468.

10. Miller WC, Corey GR, Lallinger GJ, Durack DT. International health and internal medicine residency training: the Duke University experience. Am J Med. 1995;99:291297.

11. Ramsey AH, Haq C, Gjerde CL, Rothenberg D. Career influence of an international health experience during medical school. Fam Med. Jun 2004;36(6):412-416.

12. Federico SG, Zachar PA, Oravec CM, Mandler T, Goldson E, Brown J. A successful international child health elective: The University of Colorado Department of Pediatrics' experience. Arch Pediatr Adolesc Med. February 1, 2006 2006;160(2):191-196.

13. Haq C, Rothenberg $\mathrm{D}$, Gjerde $\mathrm{C}$, et al. New world views: preparing physicians in training for global health work. Family Medicine.2000;32:566-572.

14. Sawatsky AP, Rosenman DJ, Merry SP, McDonald FS. Eight years of the Mayo international health program: What an international elective adds to resident education. Mayo Clin Proc. 2010;85:734-41.

15. Godkin M, Savageau J. The effect of medical students' international experiences on attitudes toward serving underserved multicultural Populations. Family medicine. 2003;35:273-278. 
16. Fennell $\mathrm{R}$. The impact of an international health study abroad program on university students from the United States. Glob Health Promot. 2009;16:17-23.

17. Gladding S, Zink T, Howard C, Campagna A, Slusher T, John C. International electives at the university of Minnesota global pediatric residency program: opportunities for education in all Accreditation Council for Graduate Medical Education competencies. Academic pediatrics. 2012;12:245-250.

18. Henry JA, Groen RS, Price RR, et al. The benefits of international rotations to resourcelimited settings for U.S. surgery residents. Surgery. 2013;153:445-454.

19. Gupta A, Wells CK, Horwitz RI, Bia FJ, Barry M. The international health program: the fifteen-year experience with Yale University's internal medicine residency program. The American journal of tropical medicine and hygiene. 1999;61:1019.

20. Paul A. Harris RT, Robert Thielke, Jonathon Payne, Nathaniel Gonzalez, Jose G. Conde. Research electronic data capture (REDCap) - A metadata-driven methodology and workflow process for providing translational research informatics support. J Biomed Inform. 2009;42:377-381.

21. Bhutta ZA, Chen L, Cohen J, et al. Education of health professionals for the 21st century: a global independent Commission. Lancet. 2010;375:1137-1138.

22. Kuehn BM. Guidelines, online training aim to teach physicians to weigh costs of care, become better stewards of medical resources. JAMA. 2014;311:2368-70.

23. Cooke M. Cost consciousness in patient care - what is medical education's responsibility? New England Journal of Medicine. 2010;362:1253-1255.

24. Weinberger SE. Providing high-value, cost-conscious care: a critical seventh general competency for physicians. Annals of internal medicine. 2011;155:386-388.

25. Umoren R, Stadler DJ, Gasior SL, Al-Sheikhly D, Truman B, Lowe C. Global collaboration and team-building through 3D virtual environments, Innovations in Global Medical and Health Education 2014:1 http://dx.doi.org/10.5339/igmhe.2014.1:1-10

26. Bazemore AW, Goldenhar LM, Lindsell CJ, Diller PM, Huntington MK. An international health track is associated with care for underserved US populations in subsequent clinical practice. J Grad Med Educ. 2011;3:130-137.

27. Liaw W, Bazemore A, Xierali I, Walden J, Diller P, Morikawa MJ. The association between global health training and underserved care: early findings from two longstanding tracks. Fam Med. 2013;45:263-267. 
Table 1.

Demographics of survey respondents.

\section{GHE Group ( $n=176) N \% \quad$ Control Group $(n=177) N \% \quad p$-value}

Current age (years)

$<35$

93(52.8)

$35-44$

60(34.1)

$>44$

23(13.1)

$62(35.0)$

18(10.2)

Gender

$90(51.1)$

82(46.3)

Race*

Caucasian

$90(51.1)$

$82(46.3)$

Other

142(80.7)

130(73.4)

29(16.5)

24(13.6)

NS

*Omitting unknown.

Table 2.

Univariate group comparisons of practice type and location.

\begin{tabular}{|c|c|c|}
\hline & $\begin{array}{l}\text { GHE Group } \\
(n=176) \\
\mathrm{n}(\%)\end{array}$ & $\begin{array}{l}\text { Control Group } \\
(n=177) \\
\mathrm{n}(\%)\end{array}$ \\
\hline \multicolumn{3}{|l|}{ Current Practice Type† } \\
\hline Primary Care & $50(28.4)$ & $39(22.0)$ \\
\hline Hospitalist & $36(20.5)$ & $24(13.6)$ \\
\hline Subspecialist & $79(44.9)$ & $111(62.7)$ \\
\hline Not Practicing Medicine/Research/Other & $11(6.2)$ & $3(1.7)$ \\
\hline \multicolumn{3}{|l|}{ Practice Location* } \\
\hline Urban & $80(45.5)$ & $88(49.7)$ \\
\hline Inner City & $38(21.6)$ & $30(17.0)$ \\
\hline Suburban & $34(19.3)$ & $43(24.3)$ \\
\hline Rural & $15(8.5)$ & $16(9.0)$ \\
\hline International & $9(5.1)$ & $0(0.0)$ \\
\hline \multicolumn{3}{|c|}{$\begin{array}{l}\text { Percentage of Time Spent in Global Health, Public Health, Public Policy } \\
\text { Activities† }\end{array}$} \\
\hline$<25 \%$ & 142(80.7) & 162(91.5) \\
\hline $25-49 \%$ & $19(10.8)$ & $9(5.1)$ \\
\hline$\geq 50 \%$ & $15(8.5)$ & $6(3.4)$ \\
\hline \multicolumn{3}{|l|}{ Time Spent Providing Care Outside U.S. $\ddagger$} \\
\hline$>3$ weeks/year & $18(10.3)$ & $1(0.6)$ \\
\hline 1-3 weeks/year & $19(10.8)$ & $8(4.5)$ \\
\hline$<1$ week/year & $24(13.6)$ & $31(17.5)$ \\
\hline Never & $115(65.3)$ & $137(77.4)$ \\
\hline $\begin{array}{l}* p<0.05 . \\
\dagger p<0.01 \\
\ddagger p<0.0001\end{array}$ & & \\
\hline
\end{tabular}


Table 3. Results of Multivariable Analysis

\begin{tabular}{lccc}
\hline & $\begin{array}{c}\text { Odds } \\
\text { Ratio }\end{array}$ & $95 \%$ C.I. & p-value \\
& & & \\
\hline Practice Type & 1.23 & $(0.62,2.44)$ & NS \\
$\quad$ Hospitalist vs. Primary Care & 2.20 & $(1.19,4.06)$ & 0.01 \\
$\quad$ Hospitalist vs. Subspecialist & 1.79 & $(1.06,3.03)$ & 0.03 \\
$\quad$ Primary Care vs. Subspecialist & & & \\
& 2.66 & $(1.13,6.26)$ & 0.03 \\
Cost affects choices (yes vs. no) & & & \\
& & & $<0.001$ \\
Providing care outside U.S. & 4.93 & $(1.94,12.57)$ & $<0.001$ \\
$\quad \geq 1$ week/year vs. $<1$ week/year & 4.57 & $(2.08,10.05)$ & $<0.001$ \\
$\quad \geq 1$ week/year vs. never & 0.93 & $(0.50,1.73)$ & $\mathrm{NS}$ \\
\hline 1 week/year vs. never & & &
\end{tabular}

Figure 1. Survey Respondents in Global Health Elective (GHE) and Control Groups
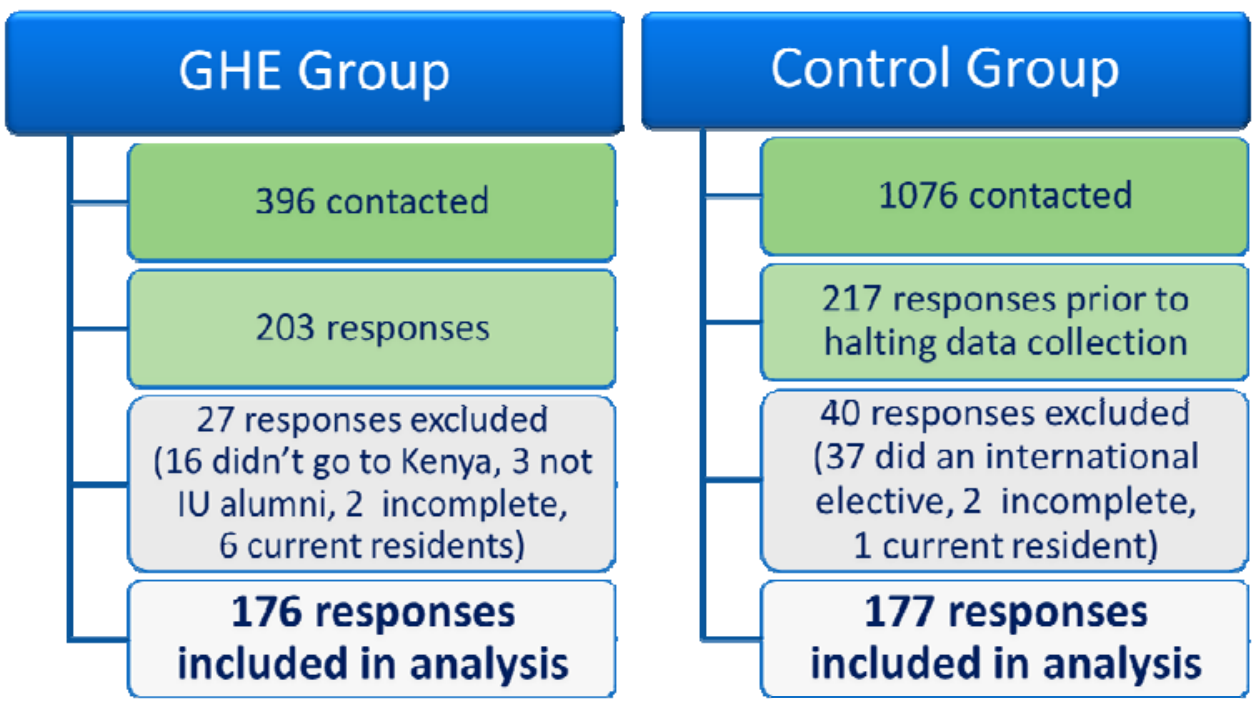